\title{
Local Torelli theorem for non-singular complete intersections
}

\author{
By Sampei UsuI \\ (Received February 9, 1976)
}

\section{§ 0. Introduction}

After studying periods of integrals of algebraic manifolds ([2]), P. A. Griffiths summarized the main results and open problems ([3]). In his article [3], he presented a problem: "(7.1). ProBlem*. Find methods to treat the local Torelli theorem. In particular, decide whether it is true or false for simply-connected canonical surfaces (i.e. surfaces with ample canonical bundle)". The local Torelli problem is the question whether a period map separates infinitely near points (see $\S 1$ ). The purpose of the present paper is to answer the above problem in the case of non-singular complete intersections with ample canonical line bundle.

In $\S 1$ we recall period map briefly in order to formulate the local Torelli problem. In $\S 2$ we give a proof of the local Torelli theorem for nonsingular complete intersections with ample canonical line bundle.

Griffiths examined the local Torelli problem in some examples such as curves, special complex manifolds (i.e. complex Kähler manifolds whose canonical line bundles are trivial), surfaces in $\boldsymbol{P}^{3}$, and surfaces on abelian varieties, and also gave some counter-examples (II, section 3 in [2]). In the investigation of hypersurfaces, Griffiths used the Macauley theorem on a polynomial ring (suggested by D. Mumford) as an essencial tool. By using the same theorem, C. Peters succeeded in giving a proof of the local Torelli theorem for cyclic branched coverings of $\boldsymbol{P}^{n}$, and of $\boldsymbol{P}^{1} \times \boldsymbol{P}^{1}$ ([6]). As for cyclic branched coverings of $\boldsymbol{P}^{n}$, since they can be seen as weighted hypersurfaces, we can go on the same route as for hypersurfaces (for "weighted complete intersection" see S. Mori [5]). In the present paper the writer shows that, for complete intersections, the Macauley theorem can be taken the place of by vanishing of some cohomology.

Throughout this paper, we understand by a variety one which is defined over the complex number field $\boldsymbol{C}$.

The writer wishes to thank C. Peters at Leiden University for the stimulating communication. 


\section{§1. Period map and local Torelli problem}

In this section we will recall the period map briefly and formulate the local Torelli problem after Griffiths (II of [2]).

Let $\left\{X_{s}\right\}_{s \in S}$ be a complex-analytic family of $m$-dimensional Kähler manifolds. Put $X=X_{0}$ for some fixed point 0 on $S$. Choose and fix a $C^{\infty}$ trivialisation $g$ of the family

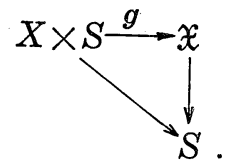

Then $g$ induces a $C^{\infty}$-isomorphism $g_{s}: X \rightarrow X_{s}$ for each point $s \in S$, and $g_{s}$ induces an isomorphism of the complex cohomology groups $g_{s}^{*}: H^{r}\left(X_{s}, C\right) \rightarrow$ $H^{r}(X, C)$. Let $H^{r}(X, C)=\sum_{p+q=r} H^{p, q}(X)$ be the Hodge decomposition. Put $H^{r}=H^{r}(X, C), h^{p, q}=\operatorname{dim}_{C} H^{p, q}(X)$, and $f^{i}=h^{r, 0}+\cdots+h^{r-i, i}(0 \leqslant i \leqslant r)$. Note that the Hodge numbers $h^{p, q}$ and hence the numbers $f^{i}$ are deformationinvariants. Let $F l\left(H^{r} ; f^{0}, \cdots, f^{r}\right)$ be the flag manifold parametrizing filtrations of $H^{r}$ by $C$-vector subspaces. We see that the mapping

$$
\Phi^{r}: S \rightarrow F l\left(H^{r} ; f^{0}, \cdots, f^{r}\right)
$$

given by

$$
\begin{array}{r}
\Phi^{r}(s)=\left[g_{s}^{*} H^{r, 0}\left(X_{s}\right) \subset g_{s}^{*}\left(H^{r, 0}\left(X_{s}\right)+H^{r-1,1}\left(X_{s}\right)\right) \subset \cdots\right. \\
\left.\subset g_{s}^{*}\left(H^{r, 0}\left(X_{s}\right)+\cdots+H^{0, r}\left(X_{s}\right)\right)\right]
\end{array}
$$

is well-defined. Griffiths showed that this map $\Phi^{r}$ is holomorphic and called it the period map in $r$-th cohomology.

On the tangent space level, we get the following factorization of $\Phi_{*}^{r}(0)$ via Kodaira-Spencer infinitesimal deformation map $\rho$ :

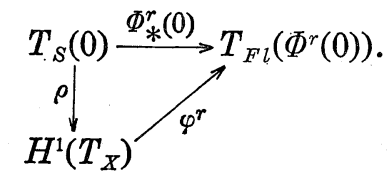

In the case that $\left\{X_{s}\right\}_{s \in S}$ is the Kuranishi family of deformations of $X, \rho$ is an isomorphism and hence it is natural to call the following the local Torelli problem.

Local Torelli problem in r-th cohomology: Is the map $\varphi^{r}$ injective?

We modify the above problem a little. Recall that

$$
T_{F l}\left(\Phi^{r}(0)\right) \approx \prod_{1 \leqslant i \leqslant r} \operatorname{Hom}\left(H^{r-i+1, i-1}(X), H^{r-i, i}(X)+\cdots+H^{0, r}(X)\right),
$$


and, in fact, the map $\varphi^{r}$ factors as follows (see Griffiths II of [2]):

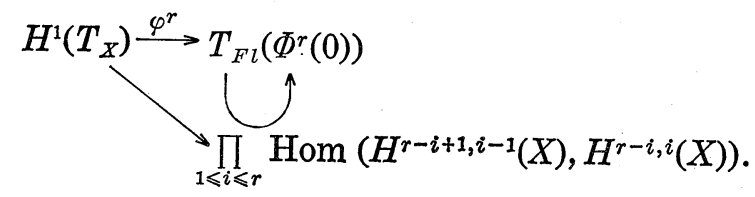

We also denote the above map $H^{1}\left(T_{X}\right) \rightarrow \prod_{1 \leqslant i \leqslant r} \operatorname{Hom}\left(H^{r-i+1, i-1}(X), H^{r-i, i}(X)\right)$ by the same notation $\varphi^{r}$. This map $\varphi^{r}$ is nothing but the map induced by the pairing $T_{X} \otimes \Omega_{X}^{r-i+1} \rightarrow \Omega_{X}^{r-i}(1 \leqslant i \leqslant r)$. Therefore the local Torelli problem in $r$-th cohomology is formulated as :

$\left(P_{0}\right) \quad$ Let $H^{1}\left(T_{X}\right) \otimes H^{r-i+1, i-1}(X) \rightarrow H^{r-i, i}(X)$ be the pairing induced by $T_{X} \otimes \Omega_{X}^{r-i+1} \rightarrow \Omega_{X}^{r-i}(1 \leqslant i \leqslant r) . \quad$ Is $\tau \in H^{1}\left(T_{X}\right)$ zero if $\tau \cdot \alpha_{i}$ is zero in $H^{r-i, i}(X)$ for every $\alpha_{i} \in H^{r-i+1, i-1}(X)(1 \leqslant i \leqslant r)$ ?

\section{§ 2. Local Torelli theorem for non-singular complete intersections}

Let $R=C\left[X_{0}, \cdots, X_{n}\right]$ be a polynomial ring over the complex number field. For each non-negative integer $a, R_{a}$ denotes a module of homogeneous forms of degree $a$. Let $a_{j}$ be integers with $a_{j}>1(1 \leqslant j \leqslant c)$ and let $F_{j} \in R_{a_{j}}$ $(1 \leqslant j \leqslant c)$. Let $I$ be the ideal of $R$ generated by $F_{1}, \cdots, F_{c}$. Suppose $X=$ $V_{+}\left(F_{1}, \cdots, F_{c}\right)$ is a non-singular complete intersection. Put $m=\operatorname{dim} X=$ $n-c$ and assume that $m \geqslant 2$. Let $K_{X}$ be the canonical line bundle of $X$ and put $k=\operatorname{deg} K_{X}=\sum_{j=1}^{c} a_{j}-(n+1)$. Assume that $k>0$.

Now we reformulate local Torelli problem for non-singular complete intersection $X$. Let $T_{X} \otimes K_{X} \widetilde{\rightarrow} \Omega_{X}^{m-1}$ be the canonical isomorphism, where $T_{X}$ is the tangent bundle of $X$ and $\Omega_{X}^{m-1}$ the $(m-1)$-th exterior product of the cotangent bundle of $X$. From this we get a homomorphism

$$
H^{1}\left(T_{X}\right) \otimes H^{0}\left(K_{X}\right) \longrightarrow H^{1}\left(\Omega_{X}^{m-1}\right)
$$

In order to prove the local Torelli theorem for $X$, it is enough to show the following:

$\left(P_{1}\right)$ The above pairing (1) is non-degenerate in the first factor.

From the well-known exact sequence for complete intersection

$$
0 \longrightarrow \mathcal{O}_{X} \longrightarrow \mathcal{O}_{X}(1)^{\oplus(n+1)} \longrightarrow T_{P} \mid X \longrightarrow 0,
$$

we get the exact sequence of cohomology groups :

$$
H^{1}\left(\mathcal{O}_{X}(1)\right)^{\oplus(n+1)} \longrightarrow H^{1}\left(T_{P} \mid X\right) \longrightarrow H^{2}\left(\mathcal{O}_{X}\right) \longrightarrow H^{2}\left(\mathcal{O}_{X}(1)\right)^{\oplus(n+1)}
$$


Since $X$ is a complete intersection of dimension $m \geqslant 2$, we see that $H^{1}\left(\mathcal{O}_{X}(1)\right)$ $=0$, and that $H^{2}\left(\mathcal{O}_{X}\right)=0$ in the case $m>2$ and $H^{2}\left(\mathcal{O}_{X}\right) \rightarrow H^{2}\left(\mathcal{O}_{X}(1)\right)^{\oplus(n+1)}$ is injective in the case $m=2$. The last statement follows from the fact that the dual of this morphism $H^{0}\left(K_{X}\right) \leftarrow H^{0}\left(K_{X}(-1)\right)^{\oplus(n+1)}$ is surjective. Hence we get $H^{1}\left(T_{P} \mid X\right)=0$. This result and another well-known exact sequence

$$
\begin{aligned}
& 0 \longrightarrow T_{X} \longrightarrow T_{P} \mid X \longrightarrow N_{X / P} \longrightarrow 0 \\
& \bigoplus_{j=1}^{c} \mathcal{O}_{X}\left(a_{j}\right)
\end{aligned}
$$

give us the following exact sequence:

$$
H^{0}\left(T_{P} \mid X\right) \longrightarrow \oplus_{j=1}^{c} H^{0}\left(\mathcal{O}_{X}\left(a_{j}\right)\right) \longrightarrow H^{1}\left(T_{X}\right) \longrightarrow 0
$$

Also from

$$
0 \longrightarrow T_{X} \otimes K_{X} \longrightarrow T_{P} \mid X \otimes K_{X} \longrightarrow N_{X / P} \otimes K_{X} \longrightarrow 0,
$$

we get

$$
H^{0}\left(T_{P}(k) \mid X\right) \longrightarrow \bigoplus_{j=1}^{c} H^{0}\left(\mathcal{O}_{X}\left(a_{j}+k\right)\right) \longrightarrow H^{1}\left(T_{X}(k)\right)
$$

Therefore $\left(P_{1}\right)$ is reformulated as:

$\left(P_{2}\right) \quad$ Let $\Phi_{j}$ be a homogeneous polynomial in $R$ of degree $a_{j}(1 \leqslant j \leqslant c)$. Suppose, for each homogeneous polynomial $G$ in $R$ of degree $k$, there exist $A_{0}, \cdots, A_{n}$ in $R$ such that

$$
G\left[\begin{array}{c}
\Phi_{1} \\
\vdots \\
\Phi_{c}
\end{array}\right] \equiv\left[\begin{array}{c}
\partial_{0} F_{1} \cdots \partial_{n} F_{1} \\
\cdots \\
\partial_{0} F_{c} \cdots \partial_{n} F_{c}
\end{array}\right]\left[\begin{array}{c}
A_{0} \\
\vdots \\
A_{n}
\end{array}\right] \quad \bmod I
$$

where $\partial_{i} F_{j}$ denotes $\partial F_{j} / \partial X_{i}(1 \leqslant j \leqslant c, 0 \leqslant i \leqslant n)$. Then there exist homogeneous polynomials $B_{0}, \cdots, B_{n}$ in $R$ such that

$$
\left[\begin{array}{c}
\Phi_{1} \\
\vdots \\
\Phi_{c}
\end{array}\right] \equiv\left[\begin{array}{c}
\partial_{0} F_{1} \cdots \partial_{n} F_{1} \\
\cdots \\
\partial_{0} F_{c} \cdots \partial_{n} F_{c}
\end{array}\right]\left[\begin{array}{c}
B_{0} \\
\vdots \\
B_{n}
\end{array}\right] \quad \bmod I .
$$

In the statement $\left(P_{2}\right)$, we may assume $G$ to be a monomial. Then, by splitting a monomial $G$ into a product of $X_{i}$ and another monomial and by multiplying it to $\Phi_{j}$ 's, we modify $\left(P_{2}\right)$ as follows (which is a little stronger assertion): 
$\left(P_{3}\right)$ Let $\Phi_{j}$ be a homogeneous polynomial in $R(1 \leqslant j \leqslant c)$. Suppose, for each $i(0 \leqslant i \leqslant n)$, there exist homogeneous polynomials $A_{i 0}, \cdots, A_{i n}$ in $R$ such that $\operatorname{deg} A_{i \nu} \leqslant k+1(0 \leqslant i, \nu \leqslant n)$ and that

$$
X_{i}\left[\begin{array}{c}
\Phi_{1} \\
\vdots \\
\Phi_{c}
\end{array}\right] \equiv\left[\begin{array}{c}
\partial_{0} F_{1} \cdots \partial_{n} F_{1} \\
\cdots \\
\partial_{0} F_{c} \cdots \partial_{n} F_{c}
\end{array}\right]\left[\begin{array}{c}
A_{i 0} \\
\vdots \\
\dot{A}_{i n}
\end{array}\right] \quad \bmod I
$$

Then there exist homogeneous polynomials $B_{0}, \cdots, B_{n}$ in $R$ such that

$$
\left[\begin{array}{c}
\Phi_{1} \\
\vdots \\
\Phi_{c}
\end{array}\right] \equiv\left[\begin{array}{c}
\partial_{0} F_{1} \cdots \partial_{n} F_{1} \\
\cdots \\
\partial_{0} F_{c} \cdots \partial_{n} F_{c}
\end{array}\right]\left[\begin{array}{c}
B_{0} \\
\vdots \\
B_{n}
\end{array}\right] \quad \bmod I .
$$

Before proving $\left(P_{3}\right)$ we prove the following lemmas.

LEMMA 1 (See Proposition 1.3 in [1]). $H^{0}\left(\Omega_{X}^{m-1}(\ell)\right)=0(\ell \leqslant 1)$.

Proof. Put $E=\oplus_{j=1}^{c} \mathcal{O}_{P}\left(-a_{j}\right)$. Let $b_{\mu}$ be non-negative integers which are not all zero. Then we have

$$
H^{q}\left(\otimes_{\mu}\left(\bigwedge^{b_{\mu}} E\right) \otimes \Omega_{P}^{p} \otimes \mathcal{O}_{P}(\ell)\right)=0 \quad \text { for } p+q<n \text { and } \ell \leqslant 1
$$

We want to show

$$
\begin{array}{ll}
H^{q}\left(\Omega_{P}^{p} \otimes \mathcal{O}_{\boldsymbol{P}}(\ell)\right) \stackrel{\sim}{\longrightarrow} H^{q}\left(\Omega_{X}^{p} \otimes \mathcal{O}_{\boldsymbol{P}}(\ell)\right) & \text { for } p+q<m=n-c, \quad \text { and } \\
H^{q}\left(\Omega_{\boldsymbol{P}}^{p} \otimes \mathcal{O}_{\boldsymbol{P}}(\ell)\right) \longrightarrow H^{q}\left(\Omega_{X}^{p} \otimes \mathcal{O}_{\boldsymbol{P}}(\ell)\right) & \text { for } p+q=m=n-c
\end{array}
$$

by induction on $p$. When $p=0$, we have $H^{0}\left(\mathcal{O}_{P}(\ell)\right) \stackrel{\sim}{\longrightarrow} H^{0}\left(\mathcal{O}_{X}(\ell)\right), H^{q}\left(\mathcal{O}_{P}(\ell)\right)$ $=0$ for $0<q \leqslant m$, and $H^{q}\left(\mathcal{O}_{X}(\ell)\right)=0$ for $0<q<m$. Now suppose that $p>0$ and that (3) is verified for $p^{\prime}<p$. The exact sequence

$$
0 \longrightarrow \check{N}_{X / P} \otimes \mathcal{O}_{P}(\ell) \longrightarrow \Omega_{P}^{1} \mid X \otimes \mathcal{O}_{P}(\ell) \longrightarrow \Omega_{X}^{1} \otimes \mathcal{O}_{P}(\ell) \longrightarrow 0
$$

induces a descending filtration of $\Omega_{P}^{p} \mid X \otimes \mathcal{O}_{P}(\ell)$ whose successive quotients are given by

$$
G r^{b}\left(\Omega_{P}^{p} \mid X \otimes \mathcal{O}_{P}(\ell)\right) \approx \bigwedge^{b} E \otimes \Omega_{X}^{p-b}
$$

The above filtration defines a spectral sequence

$$
E_{1}^{b, q-b}=H^{q}\left(\bigwedge^{b} E \otimes \Omega_{X}^{p-b} \otimes \mathcal{O}_{P}(\ell)\right) \Longrightarrow H^{q}\left(\Omega_{P}^{p} \mid X \otimes \mathcal{O}_{P}(\ell)\right) .
$$


By the induction hypothesis, we have

$$
H^{q}\left(\bigwedge^{b} E \otimes \Omega_{X}^{p-b} \otimes \mathcal{O}_{P}(\ell)\right)=0 \quad \text { for } b>0 \text { and } p+q<m+b .
$$

Hence we get

$$
\begin{array}{ll}
H^{q}\left(\Omega_{P}^{p} \mid X \otimes \mathcal{O}_{P}(\ell)\right) \stackrel{\sim}{\longrightarrow} H^{q}\left(\Omega_{X}^{p} \otimes \mathcal{O}_{P}(\ell)\right) & \text { for } p+q<m, \quad \text { and } \\
H^{q}\left(\Omega_{P}^{p} \mid X \otimes \mathcal{O}_{P}(\ell)\right) \smile H^{q}\left(\Omega_{X}^{p} \otimes \mathcal{O}_{P}(\ell)\right) & \text { for } p+q=m .
\end{array}
$$

On the other hand, since $X$ is a complete intersection, the Koszul complex of $\mathcal{O}_{X}$ gives a resolution of $\Omega_{P}^{p} \mid X \otimes \mathcal{O}_{P}(\ell)$ consisting of $\wedge^{b} E \otimes \Omega_{P}^{p} \otimes \mathcal{O}_{P}(\ell)$. This resolution defines a spectral sequence

$$
E_{1}^{-b, q}=H^{q}\left(\bigwedge^{b} E \otimes \Omega_{P}^{p} \otimes \mathcal{O}_{P}(\ell)\right) \Longrightarrow H^{q-b}\left(\Omega_{P}^{p} \mid X \otimes \mathcal{O}_{\boldsymbol{P}}(\ell)\right) .
$$

Hence by (2) we get

$$
\begin{array}{ll}
H^{q}\left(\Omega_{\boldsymbol{P}}^{p} \otimes \mathcal{O}_{\boldsymbol{P}}(\ell)\right) \stackrel{\sim}{\longrightarrow} H^{q}\left(\Omega_{\boldsymbol{P}}^{p} \mid X \otimes \mathcal{O}_{\boldsymbol{P}}(\ell)\right) & \text { for } p+q<m, \quad \text { and } \\
H^{q}\left(\Omega_{\boldsymbol{P}}^{p} \otimes \mathcal{O}_{\boldsymbol{P}}(\ell)\right) \smile H^{q}\left(\Omega_{\boldsymbol{P}}^{p} \mid X \otimes \mathcal{O}_{\boldsymbol{P}}(\ell)\right) & \text { for } p+q=m .
\end{array}
$$

Combining (4) and (5), we get (3). Applying (3) for $p=m-1$ and $q=0$, we have

$$
0=H^{0}\left(\Omega_{P}^{m-1} \otimes \mathcal{O}_{P}(\ell)\right) \stackrel{\sim}{\longrightarrow} H^{0}\left(\Omega_{X}^{m-1} \otimes \mathcal{O}_{P}(\ell)\right) \quad(\ell \leqslant 1) . \quad \text { Q.E.D. }
$$

LemMa 2. Let $A_{0}, \cdots, A_{n}$ be homogeneous polynomials in $R$ with $\operatorname{deg} A_{i} \leqslant k+2(0 \leqslant i \leqslant n)$. Suppose that

$$
\left[\begin{array}{c}
\partial_{0} F_{1} \cdots \partial_{n} F_{1} \\
\cdots \\
\partial_{0} F_{c} \cdots \partial_{n} F_{c}
\end{array}\right]\left[\begin{array}{c}
\mathrm{A}_{0} \\
\vdots \\
A_{n}
\end{array}\right] \equiv\left[\begin{array}{c}
0 \\
\vdots \\
0
\end{array}\right] \quad \bmod I .
$$

Then we can find a homogeneous polynomial $B$ in $R$, which is independent of $i$, such that

$$
A_{i} \equiv X_{i} B \bmod I(0 \leqslant i \leqslant n) .
$$

Proof. We consider the following diagram: 


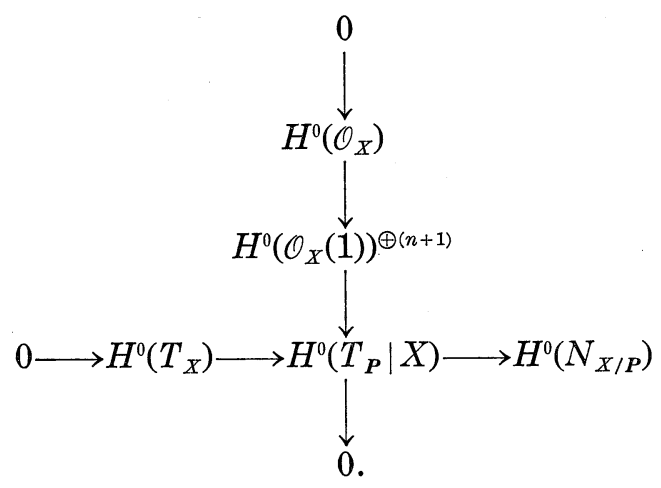

Since the homomorphism $H^{0}\left(T_{\boldsymbol{P}} \mid X\right) \rightarrow H^{0}\left(N_{X / P}\right)$ is given by

$$
\left[\begin{array}{c}
\partial_{0} F_{1} \cdots \partial_{n} F_{1} \\
\cdots \\
\partial_{0} F_{c} \cdots \partial_{n} F_{c}
\end{array}\right],
$$

the statement of Lemma 2 is equivalent to

$$
H^{0}\left(T_{X}(\ell)\right)=0 \quad(\ell \leqslant k+1) .
$$

Moreover, since $T_{X} \otimes K_{X} \widetilde{\rightarrow} \Omega_{X}^{m-1}$, that is, $T_{X}(k)=\Omega_{X}^{m-1}$, (6) is equivalent to Lemma 1.

Now we prove $\left(P_{3}\right)$.

Proof. Choose homogeneous coordinates of $P^{n}$ such that $F_{1}, \ldots, F_{c}$, $X_{0}, X_{1}, X_{2}$ form a regular sequence.

$$
\begin{aligned}
& X_{0}\left(H_{1}\right)-X_{1}\left(H_{0}\right): \\
& {\left[\begin{array}{c}
0 \\
\vdots \\
0
\end{array}\right] \equiv\left[\begin{array}{c}
\partial_{0} F_{1} \cdots \partial_{n} F_{1} \\
\cdots \\
\partial_{0} F_{c} \cdots \partial_{n} F_{c}
\end{array}\right]\left[\begin{array}{c}
X_{0} A_{10}-X_{1} A_{00} \\
\vdots \\
X_{0} A_{1 n}-X_{1} A_{0 n}
\end{array}\right] \quad \bmod I .}
\end{aligned}
$$

Applying Lemma 2 for $A_{i}=X_{0} A_{1 i}-X_{1} A_{0 i}$, we get $B$ in $R$ such that

$$
X_{0} A_{1 i}-X_{1} A_{0 i} \equiv X_{i} B \quad \bmod I \quad(0 \leqslant i \leqslant n) .
$$

Observing the above formula (7) for $i=2$ modulo $\left(F_{1}, \cdots, F_{c}, X_{0}, X_{1}\right)$, we can cancel $X_{2}$. Hence

$$
B \equiv X_{0} C+X_{1} D \quad \bmod I
$$

for some $C$ and $D$ in $R$. Applying this to the formula (7), we have 


$$
X_{0} A_{1 i}-X_{1} A_{0 i} \equiv\left(X_{0} C+X_{1} D\right) X_{i} \quad \bmod I
$$

i.e.

$$
X_{0}\left(A_{1 i}-X_{i} C\right)-X_{1}\left(A_{0 i}+X_{i} D\right) \equiv 0 \quad \bmod I .
$$

Observing this formula modulo $\left(F_{1}, \cdots, F_{c}, X_{0}\right)$, we can cancel $X_{1}$, and we have

$$
A_{0 i}+X_{i} D \equiv X_{0} E_{i} \quad \bmod I
$$

for some $E_{i}$ in $R(0 \leqslant i \leqslant n)$. Applying this formula (8) to $\left(H_{0}\right)$ in $\left(P_{3}\right)$, we have

$$
X_{0}\left\{\left[\begin{array}{c}
\Phi_{1} \\
\vdots \\
\Phi_{c}
\end{array}\right]-\left[\begin{array}{c}
\partial_{0} F_{1} \cdots \partial_{n} F_{1} \\
\cdots \\
\partial_{0} F_{c} \cdots \partial_{n} F_{c}
\end{array}\right]\left[\begin{array}{c}
E_{0} \\
\vdots \\
E_{n}
\end{array}\right]\right\} \equiv-\left[\begin{array}{c}
\partial_{0} F_{1} \cdots \partial_{n} F_{1} \\
\cdots \\
\partial_{0} F_{c} \cdots \partial_{n} F_{c}
\end{array}\right]\left[\begin{array}{c}
X_{0} \\
\vdots \\
X_{n}
\end{array}\right] D \equiv\left[\begin{array}{c}
0 \\
\vdots \\
0
\end{array}\right] \bmod I
$$

by Euler's formula. Observing this modulo $\left(F_{1}, \cdots, F_{c}\right)$, we can cancel $X_{0}$ and get the required conclusion.

Q.E.D.

\section{References}

[ 1 ] P. Deligne, Cohomologie des intersections complètes, Exp. XI in S.G.A., 7 II, Springer Lecture Notes, 340 (1973).

[2] P. A. Griffiths, Periods of integrals on algebraic manifolds I, II, III, Amer. J. Math., 90 (1968), 568-626; 805-865; Publ. Math. I.H.E.S., 38 (1970).

[ 3 ] P. A. Griffiths, Periods of integrals on algebraic manifolds: Summary of main results and discussion of open problems, Bull. Amer. Math. Soc., 75 (1970), 228-296.

[4] P. A. Griffiths and W. Schmid, Recent developments in Hodge theory: a discussion of techniques and results, Proc. Symp. at Bombey in 1973, Oxford Univ. Press (1975).

[5] S. Mori, On a generalization of complete intersections, J. Math. Kyoto Univ., 15-3 (1975), 619-646.

[6] C. Peters, The local Torelli theorem of some cyclic branched coverings, to appear.

\section{Department of Mathematics \\ KYOTO UNIVERSITY \\ 606 KYоTO-SHI}

